\title{
$N$-point free energy distribution function in one dimensional random directed polymers
}

\author{
V. Dotsenko 112 \\ ${ }^{1}$ LPTMC, Université Paris VI, 75252 Paris, France \\ 2 L.D. Landau Institute for Theoretical Physics, 119334 Moscow, Russia
}

Received April 16, 2014, in final form May 14, 2014

Explicit expression for the $N$-point free energy distribution function in one dimensional directed polymers in a random potential is derived in terms of the Bethe ansatz replica technique. The obtained result is equivalent to the one derived earlier by Prolhac and Spohn [J. Stat. Mech., 2011, P03020].

Key words: directed polymers, random potential, replicas, fluctuations, distribution function

PACS: $05.20 .-y, 75.10 . N r, 74.25 . Q t, 61.41 .+e$

\section{Introduction}

In this paper we consider the model of one-dimensional directed polymers in a quenched random potential. This model is defined in terms of an elastic string $\phi(\tau)$ directed along the $\tau$-axis within an interval $[0, t]$ which passes through a random medium described by a random potential $V(\phi, \tau)$. The energy of a given polymer's trajectory $\phi(\tau)$ is

$$
H[\phi(\tau), V]=\int_{0}^{t} \mathrm{~d} \tau\left\{\frac{1}{2}\left[\partial_{\tau} \phi(\tau)\right]^{2}+V[\phi(\tau), \tau]\right\},
$$

where the disorder potential $V[\phi, \tau]$ is described by the Gaussian distribution with a zero mean $\overline{V(\phi, \tau)}=$ 0 and the $\delta$-correlations: $\overline{V(\phi, \tau) V\left(\phi^{\prime}, \tau^{\prime}\right)}=u \delta\left(\tau-\tau^{\prime}\right) \delta\left(\phi-\phi^{\prime}\right)$ The parameter $u$ describes the strength of the disorder.

The system of this type as well as the equivalent problem of the KPZ-equation [1] describing the growth of an interface with time in the presence of noise have been the subject of intense investigations for about the last three decades (see e.g. [2-13]). Such a system exhibits numerous non-trivial features due to the interplay between elasticity and disorder. In particular, in the limit $t \rightarrow \infty$, the polymer mean squared displacement exhibits a universal scaling form $\overline{\left\langle\phi^{2}\right\rangle} \propto t^{4 / 3}$ (where $\langle\ldots\rangle$ and $\overline{(\ldots)}$ denote the thermal and the disorder averages) while the typical value of the free energy fluctuations scales as $t^{1 / 3}$. Note that in the corresponding pure system (with $V(\phi, \tau) \equiv 0)\left\langle\phi^{2}\right\rangle \propto t$ while the free energy is proportional to $\ln (t)$.

A few years ago, an exact solution for the free energy probability distribution function (PDF) has been found [14-27]. It was shown that depending on the boundary conditions, this PDF is given by the Tracy-Widom (TW) distribution [28] either of the Gaussian Unitary Ensemble (GUE) or of the Gaussian Orthogonal Ensemble (GOE) or of the Gaussian Simplectic Ensemble (GSE). Besides, recently the two-point free energy distribution function which describes the joint statistics of the free energies of the directed polymers coming to two different endpoints has been derived in [29-31]. 
For fixed boundary conditions, $\phi(0)=0 ; \phi(t)=x$, the partition function of the model (1) is

$$
Z_{t}(x)=\int_{\phi(0)=0}^{\phi(t)=x} \mathscr{D} \phi(\tau) \mathrm{e}^{-\beta H[\phi]}=\exp \left[-\beta F_{t}(x)\right]
$$

where $\beta$ is the inverse temperature and $F_{t}(x)$ is the free energy. In the limit $t \rightarrow \infty$, the free energy scales as

$$
\beta F_{t}(x)=\beta f_{0} t+\beta x^{2} / 2 t+\lambda_{t} f(x),
$$

where $f_{0}$ is the selfaveraging free energy density and

$$
\lambda_{t}=\frac{1}{2}\left(\beta^{5} u^{2} t\right)^{1 / 3} \propto t^{1 / 3} .
$$

It is the statistics of rescaled free energy fluctuations $f(x)$ which in the limit $t \rightarrow \infty$ is expected to be described by a non-trivial universal distribution $W(f)$. In fact, the first two trivial terms of this free energy can be easily eliminated by simple redefinition of the partition function:

$$
Z_{t}(x) \rightarrow \exp \left\{-\beta f_{0} t-\beta x^{2} / 2 t\right\} \tilde{Z}_{t}(x)
$$

so that

$$
\tilde{Z}_{t}(x)=\exp \left\{-\lambda_{t} f(x)\right\}
$$

The aim of the present work is to study the $N$-point free energy probability distribution function

$$
W\left(f_{1}, \ldots, f_{N} ; x_{1}, \ldots, x_{N}\right) \equiv W(\mathbf{f} ; \mathbf{x})=\lim _{t \rightarrow \infty} \operatorname{Prob}\left[f\left(x_{1}\right)>f_{1}, \ldots, f\left(x_{N}\right)>f_{N}\right],
$$

which describes the joint statistics of the free energies of $N$ directed polymers coming to $N$ different endpoints. Some time ago the result for this function has been derived in terms of the Bethe ansatz replica technique under a particular decoupling assumption [32]. Here, I am going to recompute this function using somewhat different computational tricks which do not require any supplementary assumptions and which permit to represent the final result in somewhat more explicit form.

\section{2. $N$-point distribution function}

The probability distribution function, equation (7) can be defined as follows:

$$
W(\mathbf{f} ; \mathbf{x})=\lim _{\lambda \rightarrow \infty} \sum_{L_{1}, \ldots, L_{N}=0}^{\infty} \prod_{k=1}^{N}\left[\frac{(-1)^{L_{k}}}{L_{k} !} \exp \left(\lambda L_{k} f_{k}\right)\right] \overline{\left(\prod_{k=1}^{N} \tilde{Z}_{t}\left(x_{k}\right)\right)},
$$

where $\overline{(\ldots)}$ denotes the average over random potentials. Indeed, substituting here equation (6) we get

$$
W(\mathbf{f} ; \mathbf{x})=\lim _{\lambda \rightarrow \infty} \overline{\left(\prod_{k=1}^{N} \exp \left\{-\exp \left[\lambda_{t}\left(f_{k}-f\left(x_{k}\right)\right)\right]\right\}\right)}=\overline{\left[\prod_{k=1}^{N} \theta\left(f\left(x_{k}\right)-f_{k}\right)\right]}
$$

which coincides with the definition (7).

Performing the standard averaging over random potentials in equation (8) one obtains (for details see e.g. [20])

$$
W(\mathbf{f} ; \mathbf{x})=\lim _{\lambda \rightarrow \infty} \sum_{L_{1}, \ldots, L_{N}=0}^{\infty} \prod_{k=1}^{N}\left[\frac{(-1)^{L_{k}}}{L_{k} !} \exp \left(\lambda L_{k} f_{k}\right)\right] \Psi(\underbrace{x_{1}, \ldots, x_{1}}_{L_{1}}, \underbrace{x_{2}, \ldots, x_{2}}_{L_{2}}, \ldots, \underbrace{x_{N}, \ldots, x_{N}}_{L_{N}} ; t),
$$

where the time dependent $n$-point wave function $\Psi\left(x_{1}, \ldots, x_{n} ; t\right)\left(n=\sum_{k=1}^{N} L_{k}\right)$ is the solution of the imaginary time Schrödinger equation

$$
\beta \partial_{t} \Psi(\mathbf{x} ; t)=\left[\frac{1}{2} \sum_{a=1}^{n} \partial_{x_{a}}^{2}+\frac{1}{2} \kappa \sum_{a \neq b}^{n} \delta\left(x_{a}-x_{b}\right)\right] \Psi(\mathbf{x} ; t)
$$


with $\kappa=\beta^{3} u$ and the initial condition

$$
\Psi(\mathbf{x} ; t=0)=\prod_{a=1}^{n} \delta\left(x_{a}\right) .
$$

A generic eigenstate of such a system is characterized by $n$ momenta $\left\{Q_{a}\right\}(a=1, \ldots, n)$ which split into $M(1 \leqslant M \leqslant n)$ clusters described by continuous real momenta $q_{\alpha}(\alpha=1, \ldots, M)$ and having $n_{\alpha}$ discrete imaginary parts

$$
Q_{a} \equiv q_{r}^{\alpha}=q_{\alpha}-\frac{\mathrm{i} \kappa}{2}\left(n_{\alpha}+1-2 r\right), \quad\left(r=1, \ldots, n_{\alpha}\right),
$$

with the global constraint

$$
\sum_{\alpha=1}^{M} n_{\alpha}=n
$$

The time dependent solution $\Psi(\mathbf{x}, t)$ of the Schrödinger equation 11 with the initial conditions, equation (12), can be represented in the form of a linear combination of eigenfunctions $\Psi_{\mathbf{Q}}^{(M)}(\mathbf{x})$ :

$$
\Psi(\mathbf{x} ; t)=\sum_{M=1}^{N} \frac{1}{M !} \prod_{\alpha=1}^{M}\left[\int_{-\infty}^{+\infty} \frac{\mathrm{d} q_{\alpha}}{2 \pi} \sum_{n_{\alpha}=1}^{\infty}\right] \boldsymbol{\delta}\left(\sum_{\alpha=1}^{M} n_{\alpha}, n\right) \frac{\kappa^{N}\left|C_{M}(\mathbf{q}, \mathbf{n})\right|^{2}}{N ! \prod_{\alpha=1}^{M}\left(\kappa n_{\alpha}\right)} \Psi_{\mathbf{Q}}^{(M)}(\mathbf{x}) \Psi_{\mathbf{Q}}^{(M) *}(\mathbf{0}) \exp \left\{-E_{M}(\mathbf{q}, \mathbf{n}) t\right\}
$$

Here, $\boldsymbol{\delta}(k, m)$ is the Kronecker symbol, the normalization factor

$$
\left|C_{M}(\mathbf{q}, \mathbf{n})\right|^{2}=\prod_{\alpha<\beta}^{M} \frac{\left|q_{\alpha}-q_{\beta}-\frac{\mathrm{i} \kappa}{2}\left(n_{\alpha}-n_{\beta}\right)\right|^{2}}{\left|q_{\alpha}-q_{\beta}-\frac{\mathrm{i} \kappa}{2}\left(n_{\alpha}+n_{\beta}\right)\right|^{2}}
$$

and the eigenvalues:

$$
E_{M}(\mathbf{q}, \mathbf{n})=\sum_{\alpha=1}^{M}\left(\frac{1}{2 \beta} n_{\alpha} q_{\alpha}^{2}-\frac{\kappa^{2}}{24 \beta} n_{\alpha}^{3}\right) .
$$

For a given set of integers $\left\{M ; n_{1}, \ldots, n_{M}\right\}$, the eigenfunctions $\Psi_{\mathbf{Q}}^{(M)}(\mathbf{x})$ can be represented as follows (for details see [33-37]):

$$
\Psi_{\mathbf{q}}^{(M)}(\mathbf{x})=\sum_{\mathscr{P}} \prod_{a<b}^{n}\left[1+\mathrm{i} \kappa \frac{\operatorname{sgn}\left(x_{a}-x_{b}\right)}{Q_{\mathscr{P}_{a}}-Q_{\mathscr{P}_{b}}}\right] \exp \left(\mathrm{i} \sum_{a=1}^{n} Q_{\mathscr{P}_{a}} x_{a}\right),
$$

where the summation goes over $n$ ! permutations $\mathscr{P}$ of $n$ momenta $Q_{a}$, equation (13), over $n$ particles $x_{a}$.

Substituting equations (15)-18) into equation (10) we get

$$
\begin{aligned}
& W(\mathbf{f} ; \mathbf{x})=1+\lim _{\lambda \rightarrow \infty}\left\{\sum_{L_{1}+\ldots+L_{N} \geqslant 1}^{\infty} \prod_{k=1}^{N}\left[\frac{(-1)^{L_{k}}}{L_{k} !} \exp \left(\lambda L_{k} f_{k}\right)\right]\right. \\
& \times \sum_{M=1}^{L_{1}+\ldots+L_{N}} \frac{1}{M !} \prod_{\alpha=1}^{M}\left[\sum_{n_{\alpha}=1}^{\infty} \int_{-\infty}^{+\infty} \mathrm{d} q_{\alpha} \frac{\kappa n_{\alpha}}{2 \pi \kappa n_{\alpha}} \exp \left(-\frac{t}{2 \beta} n_{\alpha} q_{\alpha}^{2}+\frac{\kappa^{2} t}{24 \beta} n_{\alpha}^{3}\right)\right] \boldsymbol{\delta}\left(\sum_{\alpha=1}^{M} n_{\alpha}, \sum_{k=1}^{N} L_{k}\right)\left|C_{M}(\mathbf{q}, \mathbf{n})\right|^{2} \\
& \left.\times \sum_{\mathscr{P}\left(L_{1}, \ldots, L_{N}\right)} \prod_{k=1}^{N}\left[\sum_{\mathscr{P}^{\left(L_{k}\right)}}\right] \prod_{k<l}^{N} \prod_{a_{k}=1}^{L_{k}} \prod_{a_{l}=1}^{L_{l}}\left(\frac{Q_{\mathscr{P}_{a_{k}}^{\left(L_{k}\right)}}-Q_{\mathscr{P}_{a_{l}}^{\left(L_{l}\right)}-\mathrm{i} \kappa}}{Q_{\mathscr{P}_{a_{k}}^{\left(L_{k}\right)}}-Q_{\mathscr{P}_{a_{l}}^{\left(L_{l}\right)}}}\right) \exp \left(\mathrm{i} \sum_{k=1}^{N} x_{k} \sum_{a_{k}=1}^{L_{k}} Q_{\mathscr{P}_{a_{k}}^{\left(L_{k}\right)}}\right)\right\} .
\end{aligned}
$$

In the above expression, the summation over permutations of $n=L_{1}+\ldots+L_{N}$ momenta $Q_{a}$ split into the internal permutations $\mathscr{P}^{\left(L_{k}\right)}$ of $L_{k}$ momenta [taken at random out of the total list $\left\{Q_{a}\right\}(a=$ $1, \ldots, n)]$ and the permutations $\mathscr{P}^{\left(L_{1}, \ldots, L_{N}\right)}$ of the momenta among the groups $L_{k}$. It is evident that due to the symmetry of the expression in equation [19], the summations over $\mathscr{P}^{\left(L_{k}\right)}$ give just the factor $L_{1}$ ! ... $L_{N}$ !. On the other hand, the structure of the Bethe ansatz wave functions, equation (18), is such that for the positions of ordered particles in the summation over permutations, the momenta $Q_{a}$ belonging to the same cluster also remain ordered (for details see e.g. [37]). Thus, in order to perform the summation over 
the permutations $\mathscr{P}^{\left(L_{1}, \ldots, L_{N}\right)}$ in equation 19 it is sufficient to split the momenta of each cluster into $N$ parts:

$$
\underbrace{\left\{q_{1}^{\alpha}, \ldots, q_{m_{\alpha}^{1}}^{\alpha}\right.}_{m_{\alpha}^{1}} ; \underbrace{q_{m_{\alpha}^{1}+1}^{\alpha}, \ldots, q_{m_{\alpha}^{1}+m_{\alpha}^{2}}^{\alpha}}_{m_{\alpha}^{2}} ; \ldots ; \underbrace{q_{\sum_{k=1}^{N-1} m_{\alpha}^{k}+1}^{\alpha}, \ldots, q_{\sum_{k=1}^{N} m_{\alpha}^{k}}^{\alpha}}_{m_{\alpha}^{N}}
$$

where the integers $m_{\alpha}^{k}=0,1, \ldots, n_{\alpha}$ are constrained by the conditions

$$
\begin{aligned}
& \sum_{k=1}^{N} m_{\alpha}^{k}=n_{\alpha} \\
& \sum_{\alpha=1}^{M} m_{\alpha}^{k}=L_{k}
\end{aligned}
$$

and the momenta of every group $\left\{q_{\sum_{l=1}^{k-1} m_{\alpha}^{l}+1}^{\alpha}, \ldots, q_{\sum_{l=1}^{k} m_{\alpha}^{l}}^{\alpha}\right\}$ all belong to the particles whose coordinates are all equal to $x_{k}$. Let us redefine:

$$
q_{\sum_{l=1}^{k-1} m_{\alpha}^{l}+r}^{\alpha} \equiv q_{k, r}^{\alpha}=q_{\alpha}+\frac{\mathrm{i} \kappa}{2}\left(n_{\alpha}+1-2 \sum_{l=1}^{k-1} m_{\alpha}^{l}-2 r\right)
$$

In this way, the summation over $\mathscr{P}^{\left(L_{1}, \ldots, L_{N}\right)}$ is changed by the summation over the integers $\left\{m_{\alpha}^{k}\right\}$. Substituting equations (20)-(23) into equation (19) after simple algebra, we find

$$
\begin{aligned}
W(\mathbf{f} ; \mathbf{x})= & 1+\lim _{\lambda \rightarrow \infty}\left(\sum _ { M = 1 } ^ { \infty } \frac { ( - 1 ) ^ { M } } { M ! } \prod _ { \alpha = 1 } ^ { M } \left\{\sum_{\sum_{k}^{N} m_{\alpha}^{k} \geqslant 1}(-1)^{\sum_{k}^{N} m_{\alpha}^{k}-1} \int_{-\infty}^{+\infty} \frac{\mathrm{d} q_{\alpha}}{2 \pi \kappa\left(\sum_{k}^{N} m_{\alpha}^{k}\right)}\right.\right. \\
& \left.\times \exp \left[\lambda \sum_{k=1}^{N} m_{\alpha}^{k} f_{k}+\mathrm{i} \sum_{k=1}^{N} m_{\alpha}^{k} x_{k} q_{\alpha}-\frac{1}{4} \kappa \sum_{k, l=1}^{N} m_{\alpha}^{k} m_{\alpha}^{l}\left|x_{k}-x_{l}\right|-\frac{t}{2 \beta} q_{\alpha}^{2} \sum_{k=1}^{N} m_{\alpha}^{k}+\frac{\kappa^{2} t}{24 \beta}\left(\sum_{k=1}^{N} m_{\alpha}^{k}\right)^{3}\right]\right\} \\
& \left.\times\left|C_{M}\left(\mathbf{q} ;\left\{m_{\alpha}^{k}\right\}\right)\right|^{2} G_{M}\left(\mathbf{q} ;\left\{m_{\alpha}^{k}\right\}\right)\right],
\end{aligned}
$$

where the normalization constant $\left|C_{M}\left(\mathbf{q} ;\left\{m_{\alpha}^{k}\right\}\right)\right|^{2}$ is given in equation (16) (with $\left.n_{\alpha}=\sum_{k=1}^{N} m_{\alpha}^{k}\right)$ and

$$
G_{M}\left(\mathbf{q} ;\left\{m_{\alpha}^{k}\right\}\right)=\prod_{\alpha=1}^{M} \prod_{k<l}^{N} \prod_{r=1}^{m_{\alpha}^{k}} \prod_{r^{\prime}=1}^{m_{\alpha}^{l}}\left(\frac{q_{k, r}^{\alpha}-q_{l, r^{\prime}}^{\alpha}-\mathrm{i} \kappa}{q_{k, r}^{\alpha}-q_{l, r^{\prime}}^{\alpha}}\right) \prod_{\alpha<\beta}^{M} \prod_{k=1}^{N} \prod_{l=1}^{N} \prod_{r=1}^{m_{\alpha}^{k}} \prod_{r^{\prime}=1}^{m_{\alpha}^{l}}\left(\frac{q_{k, r}^{\alpha}-q_{l, r^{\prime}}^{\alpha}-\mathrm{i} \kappa}{q_{k, r}^{\alpha}-q_{l, r^{\prime}}^{\alpha}}\right)
$$

Substituting the expressions for $q_{k, r}^{\alpha}$, equation [23, one can find an explicit formula for the above factor $G_{M}$ which is rather cumbersome: it contains the products of all kinds of the Gamma functions of the type $\Gamma\left[1+\frac{1}{2}\left(\sum_{k}^{N}( \pm) m_{\alpha}^{k}+\sum_{l}^{N}( \pm) m_{\beta}^{l}\right) \pm \frac{1}{\kappa}\left(q_{\alpha}-q_{\beta}\right)\right]$ [the example of this kind of the product is given in [38], equation (A17)]. We do not reproduce it here as it turns out to be irrelevant in the limit $t \rightarrow \infty$ (see below).

After rescaling

$$
\begin{aligned}
& q_{\alpha} \rightarrow \frac{\kappa}{2 \lambda} q_{\alpha} \\
& x_{k} \rightarrow \frac{2 \lambda^{2}}{\kappa} x_{k}
\end{aligned}
$$

with

$$
\lambda=\frac{1}{2}\left(\frac{\kappa^{2} t}{\beta}\right)^{1 / 3}=\frac{1}{2}\left(\beta^{5} u^{2} t\right)^{1 / 3}
$$


the normalization factor $\mid C_{M}\left(\mathbf{q} ;\left.\left\{m_{\alpha}^{k}\right\}\right|^{2}\right.$, equation (16) (with $n_{\alpha}=\sum_{k}^{N} m_{\alpha}^{k}$ ), can be represented as follows:

$$
\begin{aligned}
\left|C_{M}\left(\mathbf{q} ;\left\{m_{\alpha}^{k}\right\}\right)\right|^{2} & =\prod_{\alpha<\beta}^{M} \frac{\left|\lambda \sum_{k}^{N} m_{\alpha}^{k}-\lambda \sum_{k}^{N} m_{\beta}^{k}-\mathrm{i} q_{\alpha}+\mathrm{i} q_{\beta}\right|^{2}}{\lambda \sum_{k}^{N} m_{\alpha}^{k}+\lambda \sum_{k}^{N} m_{\beta}^{k}-\mathrm{i} q_{\alpha}+\left.\mathrm{i} q_{\beta}\right|^{2}} \\
& =\left[\prod_{\alpha=1}^{M}\left(2 \lambda \sum_{k}^{N} m_{\alpha}^{k}\right)\right] \operatorname{det}\left[\frac{1}{\left(\sum_{k}^{N} \lambda m_{\alpha}^{k}-\mathrm{i} q_{\alpha}\right)+\left(\sum_{k}^{N} \lambda m_{\beta}^{k}+\mathrm{i} q_{\beta}\right)}\right]_{\alpha, \beta=1, \ldots, M} .
\end{aligned}
$$

Substituting equation (25)-(28) into equation (23) and using the Airy function relation

$$
\exp \left[\frac{1}{3} \lambda^{3}\left(\sum_{k}^{N} m_{\alpha}^{k}\right)^{3}\right]=\int_{-\infty}^{+\infty} \mathrm{d} y \operatorname{Ai}(y) \exp \left[\lambda\left(\sum_{k}^{N} m_{\alpha}^{k}\right) y\right]
$$

we get

$$
\begin{aligned}
W(\mathbf{f} ; \mathbf{x})= & 1+\lim _{\lambda \rightarrow \infty}\left(\sum _ { M = 1 } ^ { \infty } \frac { ( - 1 ) ^ { M } } { M ! } \prod _ { \alpha = 1 } ^ { M } \left\{\iint_{-\infty}^{+\infty} \frac{\mathrm{d} q_{\alpha} \mathrm{d} y_{\alpha}}{2 \pi} \operatorname{Ai}\left(y_{\alpha}+q_{\alpha}^{2}\right)\right.\right. \\
& \left.\times \sum_{\sum_{k}^{N} m_{\alpha}^{k} \geqslant 1}(-1)^{\sum_{k}^{N} m_{\alpha}^{k}-1} \exp \left[\lambda \sum_{k=1}^{N} m_{\alpha}^{k}\left(y_{\alpha}+f_{k}+\mathrm{i} x_{k} q_{\alpha}\right)-\frac{1}{2} \lambda^{2} \sum_{k, l=1}^{N} m_{\alpha}^{k} m_{\alpha}^{l} \Delta_{k l}\right]\right\} \\
& \left.\times \operatorname{det} \hat{K}\left[\left(\sum_{k}^{N} \lambda m_{\alpha}^{k}, q_{\alpha}\right) ;\left(\sum_{k}^{N} \lambda m_{\beta}^{k}, q_{\beta}\right)\right]_{\alpha, \beta=1, \ldots, M} G_{M}\left(\frac{\kappa \mathbf{q}}{2 \lambda} ;\left\{m_{\alpha}^{k}\right\}\right)\right)
\end{aligned}
$$

where

$$
\Delta_{k l}=\left|x_{k}-x_{l}\right|
$$

and

$$
\hat{K}\left[\left(\sum_{k}^{N} \lambda m_{\alpha}^{k}, q_{\alpha}\right) ;\left(\sum_{k}^{N} \lambda m_{\beta}^{k}, q_{\beta}\right)\right]=\frac{1}{\left(\sum_{k}^{N} \lambda m_{\alpha}^{k}-\mathrm{i} q_{\alpha}\right)+\left(\sum_{k}^{N} \lambda m_{\beta}^{k}+\mathrm{i} q_{\beta}\right)} .
$$

The quadratic in $m_{\alpha}^{k}$ term in the exponential of equation (31) can be linearized as follows:

$$
\begin{aligned}
\exp \left\{-\frac{1}{2} \lambda^{2} \sum_{k, l=1}^{N} m_{\alpha}^{k} m_{\alpha}^{l} \Delta_{k l}\right\}= & \exp \left\{-\frac{1}{4} \lambda^{2} \sum_{k, l=1}^{N} \Delta_{k l}\left(m_{\alpha}^{k}+m_{\alpha}^{l}\right)^{2}+\frac{1}{2} \lambda^{2} \sum_{k=1}^{N}\left(m_{\alpha}^{k}\right)^{2} \sum_{l=1}^{N} \Delta_{k l}\right\} \\
= & \prod_{k, l=1}^{N}\left\{\int_{-\infty}^{+\infty} \frac{\mathrm{d} \xi_{k l}^{\alpha}}{\sqrt{2 \pi}} \exp \left[-\frac{1}{2}\left(\xi_{k l}^{\alpha}\right)^{2}\right]\right\} \prod_{k=1}^{N}\left\{\int_{-\infty}^{+\infty} \frac{\mathrm{d} \eta_{k}^{\alpha}}{\sqrt{2 \pi}} \exp \left[-\frac{1}{2}\left(\eta_{k}^{\alpha}\right)^{2}\right]\right\} \\
& \times \exp \left\{\lambda \sum_{k}^{N}\left[\frac{\mathrm{i}}{\sqrt{2}} \sum_{l=1}^{N} \sqrt{\Delta_{k l}}\left(\xi_{k l}^{\alpha}+\xi_{l k}^{\alpha}\right)-\sqrt{\gamma_{k}} \eta_{k}^{\alpha}\right] m_{\alpha}^{k}\right\}
\end{aligned}
$$

where

$$
\gamma_{k}=\sum_{l=1}^{N} \Delta_{k l}=\sum_{l=1}^{N}\left|x_{k}-x_{l}\right| .
$$

Substituting the representation (34) into equation (31) and redefining the integration parameters

$$
\eta_{k}^{\alpha} \rightarrow \eta_{k}^{\alpha}+\frac{\mathrm{i}}{\sqrt{\gamma_{k}}} q_{\alpha} x_{k}+\mathrm{i} \sum_{l=1}^{N} \sqrt{\frac{\Delta_{k l}}{2 \gamma_{k}}}\left(\xi_{k l}^{\alpha}+\xi_{l k}^{\alpha}\right)
$$


we get

$$
\begin{aligned}
W(\mathbf{f} ; \mathbf{x})= & +\sum_{M=1}^{\infty} \frac{(-1)^{M}}{M !} \prod_{\alpha=1}^{M}\left(\iint_{-\infty}^{+\infty} \frac{\mathrm{d} q_{\alpha} \mathrm{d} y_{\alpha}}{2 \pi} \operatorname{Ai}\left(y_{\alpha}+q_{\alpha}^{2}\right) \prod_{k, l=1}^{N}\left(\int_{-\infty}^{+\infty} \frac{\mathrm{d} \xi_{k l}^{\alpha}}{\sqrt{2 \pi}}\right) \prod_{k=1}^{N} \int_{-\infty}^{+\infty} \frac{\mathrm{d} \eta_{k}^{\alpha}}{\sqrt{2 \pi}}\right) \\
& \left.\times \exp \left\{-\frac{1}{2} \sum_{k, l=1}^{N}\left(\xi_{k l}^{\alpha}\right)^{2}-\frac{1}{2} \sum_{k=1}^{N}\left[\eta_{k}^{\alpha}+\frac{\mathrm{i}}{\sqrt{\gamma_{k}}} q_{\alpha} x_{k}+\mathrm{i} \sum_{l=1}^{N} \sqrt{\frac{\Delta_{k l}}{2 \gamma_{k}}}\left(\xi_{k l}^{\alpha}+\xi_{l k}^{\alpha}\right)\right]^{2}\right\}\right) \mathscr{S}\left(\mathbf{f}, \mathbf{y}, \mathbf{q},\left\{\eta_{k}\right\}\right),
\end{aligned}
$$

where

$$
\begin{aligned}
\mathscr{S}\left(\mathbf{f}, \mathbf{y}, \mathbf{q},\left\{\eta_{k}\right\}\right)= & \lim _{\lambda \rightarrow \infty} \prod_{\alpha=1}^{M}\left\{\sum_{\sum_{k}^{N} m_{\alpha}^{k} \geqslant 1}(-1)^{\sum_{k}^{N} m_{\alpha}^{k}-1} \exp \left[\lambda \sum_{k=1}^{N} m_{\alpha}^{k}\left(y_{\alpha}+f_{k}-\sqrt{\gamma_{k}} \eta_{k}\right)\right]\right. \\
& \left.\times \operatorname{det} \hat{K}\left[\left(\sum_{k}^{N} \lambda m_{\alpha}^{k}, q_{\alpha}\right) ;\left(\sum_{k}^{N} \lambda m_{\beta}^{k}, q_{\beta}\right)\right]_{\alpha, \beta=1, \ldots, M} G_{M}\left(\frac{\kappa \mathbf{q}}{2 \lambda} ;\left\{m_{\alpha}^{k}\right\}\right)\right\} .
\end{aligned}
$$

The summations over $m_{\alpha}^{k}$ in the above expression can be performed as follows:

$$
\begin{aligned}
\mathscr{S}\left(\mathbf{f}, \mathbf{y}, \mathbf{q},\left\{\eta_{k}\right\}\right)= & \lim _{\lambda \rightarrow \infty} \prod_{\alpha=1}^{M}\left[\prod_{k=1}^{N}\left(\sum_{m_{\alpha}^{k}=0}^{\infty} \delta_{m_{\alpha}^{k}, 0}\right)-(-1)^{N} \prod_{k=1}^{N}\left\{\sum_{m_{\alpha}^{k}=0}^{\infty}(-1)^{m_{\alpha}^{k}-1} \exp \left[\lambda m_{\alpha}^{k}\left(y_{\alpha}+f_{k}-\sqrt{\gamma_{k}} \eta_{k}\right)\right]\right\}\right] \\
& \times \operatorname{det} \hat{K}\left[\left(\sum_{k}^{N} \lambda m_{\alpha}^{k}, q_{\alpha}\right) ;\left(\sum_{k}^{N} \lambda m_{\beta}^{k}, q_{\beta}\right)\right]_{\alpha, \beta=1, \ldots, M} \times G_{M}\left(\frac{\kappa \mathbf{q}}{2 \lambda} ;\left\{m_{\alpha}^{k}\right\}\right) \\
= & \lim _{\lambda \rightarrow \infty} \prod_{\alpha=1}^{M}\left[\prod_{k=1}^{N}\left(\int_{\mathscr{C}} \mathrm{d} z_{\alpha}^{k} \delta\left(z_{\alpha}^{k}\right)\right)-(-1)^{N} \prod_{k=1}^{N}\left\{\int_{\mathscr{C}} \frac{\mathrm{d} z_{\alpha}^{k}}{2 \mathrm{i} \sin \left(\pi z_{\alpha}^{k}\right)} \exp \left[\lambda z_{\alpha}^{k}\left(y_{\alpha}+f_{k}-\sqrt{\gamma_{k}} \eta_{k}\right)\right]\right\}\right] \\
& \times \operatorname{det} \hat{K}\left[\left(\sum_{k}^{N} \lambda z_{\alpha}^{k}, q_{\alpha}\right) ;\left(\sum_{k}^{N} \lambda z_{\beta}^{k}, q_{\beta}\right)\right]_{\alpha, \beta=1, \ldots, M} G_{M}\left(\frac{\kappa \mathbf{q}}{2 \lambda} ;\left\{z_{\alpha}^{k}\right\}\right),
\end{aligned}
$$

where the integration goes over the contour $\mathscr{C}$ shown in figure 1 Redefining $z_{\alpha}^{k} \rightarrow z_{\alpha}^{k} / \lambda$, in the limit

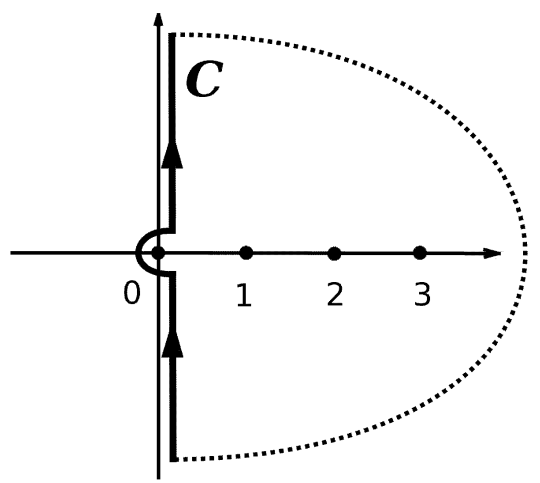

Figure 1. The contours of integration in the complex plane used for summing the series equation 39. 
$\lambda \rightarrow \infty$, we get

$$
\begin{aligned}
\mathscr{S}\left(\mathbf{f}, \mathbf{y}, \mathbf{q},\left\{\eta_{k}\right\}\right)= & \prod_{\alpha=1}^{M}\left[\prod_{k=1}^{N}\left(\int_{\mathscr{C}} \mathrm{d} z_{\alpha}^{k} \delta\left(z_{\alpha}^{k}\right)\right)-(-1)^{N} \prod_{k=1}^{N}\left\{\int_{\mathscr{C}} \frac{\mathrm{d} z_{\alpha}^{k}}{2 \pi \mathrm{i} z_{\alpha}^{k}} \exp \left[z_{\alpha}^{k}\left(y_{\alpha}+f_{k}-\sqrt{\gamma_{k}} \eta_{k}\right)\right]\right\}\right] \\
& \times \operatorname{det} \hat{K}\left[\left(\sum_{k}^{N} z_{\alpha}^{k}, q_{\alpha}\right) ;\left(\sum_{k}^{N} z_{\beta}^{k}, q_{\beta}\right)\right]_{\alpha, \beta=1, \ldots, M} \lim _{\lambda \rightarrow \infty} G_{M}\left(\frac{\kappa \mathbf{q}}{2 \lambda} ;\left\{\frac{z_{\alpha}^{k}}{\lambda}\right\}\right) .
\end{aligned}
$$

Taking into account the Gamma function property $\lim _{|z| \rightarrow 0} \Gamma(1+z)=1$, one can easily demonstrate (see e.g. [38]) that

$$
\lim _{\lambda \rightarrow \infty} G_{M}\left(\frac{\kappa \mathbf{q}}{2 \lambda} ;\left\{\frac{z_{\alpha}^{k}}{\lambda}\right\}\right)=1
$$

Thus, in the limit $\lambda \rightarrow \infty$, the expression (37) takes the form of the Fredholm determinant

$$
\begin{aligned}
W(\mathbf{f} ; \mathbf{x})= & \left.1+\sum_{M=1}^{\infty} \frac{(-1)^{M}}{M !} \prod_{\alpha=1}^{M}\left[\iint_{-\infty}^{+\infty} \frac{\mathrm{d} q_{\alpha} \mathrm{d} y_{\alpha}}{2 \pi} \operatorname{Ai}\left(y_{\alpha}+q_{\alpha}^{2}\right) \prod_{k, l=1}^{N} \int_{-\infty}^{+\infty} \frac{\mathrm{d} \xi_{k l}^{\alpha}}{\sqrt{2 \pi}}\right) \prod_{k=1}^{N} \int_{-\infty}^{+\infty} \frac{\mathrm{d} \eta_{k}^{\alpha}}{\sqrt{2 \pi}}\right) \\
& \times \exp \left\{-\frac{1}{2} \sum_{k, l=1}^{N} \xi_{k l}^{2}-\frac{1}{2} \sum_{k=1}^{N}\left[\eta_{k}^{\alpha}+\frac{\mathrm{i}}{\sqrt{\gamma_{k}}} q_{\alpha} x_{k}+\mathrm{i} \sum_{l=1}^{N} \sqrt{\frac{\Delta_{k l}}{2 \gamma_{k}}}\left(\xi_{k l}^{\alpha}+\xi_{l k}^{\alpha}\right)\right]^{2}\right\} \\
& \left.\times \prod_{k=1}^{N}\left[\int_{\mathscr{C}} \mathrm{d} z_{\alpha}^{k}\right)\left\{\prod_{k=1}^{N} \delta\left(z_{\alpha}^{k}\right)-(-1)^{N} \prod_{k=1}^{N} \frac{1}{2 \pi \mathrm{i} z_{\alpha}^{k}} \exp \left[z_{\alpha}^{k}\left(y_{\alpha}+f_{k}-\sqrt{\gamma_{k}} \eta_{k}\right)\right]\right\}\right] \\
& \times \operatorname{det} \hat{K}\left[\left(\sum_{k}^{N} z_{\alpha}^{k}, q_{\alpha}\right) ;\left(\sum_{k}^{N} z_{\beta}^{k}, q_{\beta}\right)\right]_{\alpha, \beta=1, \ldots, M} \\
\equiv & \operatorname{det}[\hat{1}-\hat{A}]=\exp \left\{-\sum_{M=1}^{\infty} \frac{1}{M} \operatorname{Tr} \hat{A}^{M}\right\},
\end{aligned}
$$

where $\hat{A}$ is the integral operator with the kernel

$$
\begin{aligned}
A\left[\left(\sum_{k}^{N} z^{k}, q\right) ;\left(\sum_{k}^{N} \tilde{z}^{k}, \tilde{q}\right)\right]= & \int_{-\infty}^{+\infty} \frac{\mathrm{d} y}{2 \pi} \operatorname{Ai}\left(y+q^{2}\right) \prod_{k, l=1}^{N}\left(\int_{-\infty}^{+\infty} \frac{\mathrm{d} \xi_{k l}}{\sqrt{2 \pi}}\right) \prod_{k=1}^{N}\left(\int_{-\infty}^{+\infty} \frac{\mathrm{d} \eta_{k}}{\sqrt{2 \pi}}\right) \\
& \times \exp \left\{-\frac{1}{2} \sum_{k, l=1}^{N} \xi_{k l}^{2}-\frac{1}{2} \sum_{k=1}^{N}\left[\eta_{k}+\frac{\mathrm{i}}{\sqrt{\gamma_{k}}} q_{\alpha} x_{k}+\mathrm{i} \sum_{l=1}^{N} \sqrt{\frac{\Delta_{k l}}{2 \gamma_{k}}}\left(\xi_{k l}+\xi_{l k}\right)\right]^{2}\right\} \\
& \times\left\{\prod_{k=1}^{N} \delta\left(z^{k}\right)-(-1)^{N} \prod_{k=1}^{N} \frac{1}{2 \pi \mathrm{i} z^{k}} \exp \left[z^{k}\left(y+f_{k}-\sqrt{\gamma_{k}} \eta_{k}\right)\right]\right\} \\
& \times \frac{1}{\sum_{k}^{N} z^{k}-\mathrm{i} q+\sum_{k}^{N} \tilde{z}^{k}+\mathrm{i} \tilde{q}} .
\end{aligned}
$$

Correspondingly, for the trace of this operator in the $M$-th power [in the exponential representation of 
the Fredholm determinant, equation [43] we get

$$
\begin{aligned}
\operatorname{Tr} \hat{A}^{M}= & \prod_{\alpha=1}^{M}\left[\iint_{-\infty}^{+\infty} \frac{\mathrm{d} y \mathrm{~d} q_{\alpha}}{2 \pi} \mathrm{Ai}\left(y+q_{\alpha}^{2}\right) \prod_{k, l=1}^{N}\left(\int_{-\infty}^{+\infty} \frac{\mathrm{d} \xi_{k l}}{\sqrt{2 \pi}}\right) \prod_{k=1}^{N}\left(\int_{-\infty}^{+\infty} \frac{\mathrm{d} \eta_{k}}{\sqrt{2 \pi}}\right)\right. \\
& \times \exp \left\{-\frac{1}{2} \sum_{k, l=1}^{N} \xi_{k l}^{2}-\frac{1}{2} \sum_{k=1}^{N}\left[\eta_{k}+\frac{i}{\sqrt{\gamma_{k}}} q_{\alpha} x_{k}+\mathrm{i} \sum_{l=1}^{N} \sqrt{\frac{\Delta_{k l}}{2 \gamma_{k}}}\left(\xi_{k l}+\xi_{l k}\right)\right]^{2}\right\} \\
& \left.\times \prod_{k=1}^{N}\left(\int_{\mathscr{C}} \mathrm{d} z_{\alpha}^{k}\right)\left\{\prod_{k=1}^{N} \delta\left(z_{\alpha}^{k}\right)-(-1)^{N} \prod_{k=1}^{N} \frac{1}{2 \pi \mathrm{i} z_{\alpha}^{k}} \exp \left[z_{\alpha}^{k}\left(y+f_{k}-\sqrt{\gamma_{k}} \eta_{k}\right)\right]\right\}\right] \\
& \times \prod_{\alpha=1}^{M}\left(\frac{1}{\sum_{k}^{N} z_{\alpha}^{k}-\mathrm{i} q_{\alpha}+\sum_{k}^{N} z_{\alpha+1}^{k}+\mathrm{i} q_{\alpha+1}}\right)
\end{aligned}
$$

where, by definition, $z_{M+1}^{k} \equiv z_{1}^{k}$ and $q_{M+1} \equiv q_{1}$.

Substituting

$$
\frac{1}{\sum_{k}^{N} z_{\alpha}^{k}-\mathrm{i} q_{\alpha}+\sum_{k}^{N} z_{\alpha+1}^{k}+\mathrm{i} q_{\alpha+1}}=\int_{0}^{\infty} \mathrm{d} \omega_{\alpha} \exp \left\{-\omega_{\alpha}\left(\sum_{k}^{N} z_{\alpha}^{k}-\mathrm{i} q_{\alpha}+\sum_{k}^{N} z_{\alpha+1}^{k}+\mathrm{i} q_{\alpha+1}\right)\right\}
$$

into equation 45 we obtain

$$
\operatorname{Tr} \hat{A}^{M}=\int_{0}^{\infty} \ldots \int_{0}^{\infty} \mathrm{d} \omega_{1} \ldots \mathrm{d} \omega_{M} \prod_{\alpha=1}^{M} A\left(\omega_{\alpha} ; \omega_{\alpha+1}\right)
$$

where

$$
\begin{aligned}
A\left(\omega ; \omega^{\prime}\right)= & \iint_{-\infty}^{+\infty} \frac{\mathrm{d} y \mathrm{~d} q}{2 \pi} \operatorname{Ai}\left(y+q^{2}+\omega+\omega^{\prime}\right) \prod_{k, l=1}^{N}\left(\int_{-\infty}^{+\infty} \frac{\mathrm{d} \xi_{k l}}{\sqrt{2 \pi}}\right) \prod_{k=1}^{N}\left(\int_{-\infty}^{+\infty} \frac{\mathrm{d} \eta_{k}}{\sqrt{2 \pi}}\right) \\
& \times \exp \left\{-\frac{1}{2} \sum_{k, l=1}^{N} \xi_{k l}^{2}-\frac{1}{2} \sum_{k=1}^{N}\left[\eta_{k}+\frac{\mathrm{i}}{\sqrt{\gamma_{k}}} q x_{k}+\mathrm{i} \sum_{l=1}^{N} \sqrt{\frac{\Delta_{k l}}{2 \gamma_{k}}}\left(\xi_{k l}+\xi_{l k}\right)\right]^{2}-\mathrm{i} q\left(\omega-\omega^{\prime}\right)\right\} \\
& \times\left\{1-(-1)^{N} \prod_{k=1}^{N} \int_{\mathscr{C}} \frac{\mathrm{d} z^{k}}{2 \pi \mathrm{i} z^{k}} \exp \left[z^{k}\left(y+f_{k}-\sqrt{\gamma_{k}} \eta_{k}\right)\right]\right\}
\end{aligned}
$$

Integrating over $z^{1}, \ldots, z^{N}$, we finally get

$$
\begin{aligned}
A\left(\omega ; \omega^{\prime}\right)= & \iint_{-\infty}^{+\infty} \frac{\mathrm{d} y \mathrm{~d} q}{2 \pi} \operatorname{Ai}\left(y+q^{2}+\omega+\omega^{\prime}\right) \prod_{k, l=1}^{N}\left(\int_{-\infty}^{+\infty} \frac{\mathrm{d} \xi_{k l}}{\sqrt{2 \pi}}\right) \prod_{k=1}^{N}\left(\int_{-\infty}^{+\infty} \frac{\mathrm{d} \eta_{k}}{\sqrt{2 \pi}}\right) \\
& \times \exp \left\{-\frac{1}{2} \sum_{k, l=1}^{N} \xi_{k l}^{2}-\frac{1}{2} \sum_{k=1}^{N}\left[\eta_{k}+\frac{\mathrm{i}}{\sqrt{\gamma_{k}}} q x_{k}+\mathrm{i} \sum_{l=1}^{N} \sqrt{\frac{\Delta_{k l}}{2 \gamma_{k}}}\left(\xi_{k l}+\xi_{l k}\right)\right]^{2}-\mathrm{i} q\left(\omega-\omega^{\prime}\right)\right\} \\
& \times\left[1-(-1)^{N} \prod_{k=1}^{N} \theta\left(-y-f_{k}+\eta_{k} \sqrt{\gamma_{k}}\right)\right]
\end{aligned}
$$

where $\Delta_{k l}=\left|x_{k}-x_{l}\right|$ and $\gamma_{k}=\sum_{l=1}^{N} \Delta_{k l}$.

Thus, the $N$-point free energy distribution function $W\left(f_{1}, \ldots, f_{N} ; x_{1}, \ldots, x_{N}\right)$, equation (7), is given by the Fredholm determinant

$$
W(\mathbf{f} ; \mathbf{x})=\operatorname{det}[\hat{1}-\hat{A}],
$$

where $\hat{A}$ is the integral operator with the kernel $A\left(\omega ; \omega^{\prime}\right)$ (with $\omega, \omega^{\prime} \geqslant 0$ ) represented in equation 49 . 


\section{Conclusions}

In this paper using the method developed in [30] we extended our result to the spatial $N$-point free energy distribution function in the thermodynamic limit $t \rightarrow \infty$. It should be noted that following the ideas of the proof [31] for the two-point function, one can easily demonstrate that the result [49)-(50) obtained in this paper is equivalent to that derived earlier by Prolhac and Spohn [32]. It should be stressed, however, that since the obtained result for the kernel $A\left(\omega ; \omega^{\prime}\right)$, equation 49 , has a rather complicated structure, its analytic properties are at present completely unclear and their study would require special efforts.

\section{Acknowledgements}

This work was supported in part by the grant IRSES DCPA PhysBio-269139.

\section{References}

1. Kardar M., Parisi G., Zhang Y.-C., Phys. Rev. Lett., 1986, 56, 889; doi 10.1103/PhysRevLett.56.889

2. Halpin-Healy T., Zhang Y.-C., Phys. Rep., 1995, 254, 215; doi 10.1016/0370-1573(94)00087-J.

3. Burgers J.M., The Nonlinear Diffusion Equation, Reidel, Dordrecht, 1974.

4. Kardar M., Statistical Physics of Fields, Cambridge University Press, Cambridge, 2007.

5. Huse D.A., Henley C.L., Fisher D.S., Phys. Rev. Lett., 1985, 55, 2924; doi 10.1103/PhysRevLett.55.2924

6. Huse D.A., Henley C.L., Phys. Rev. Lett., 1985, 54, 2708; doi $10.1103 /$ PhysRevLett.54.2708

7. Kardar M., Zhang Y.-C., Phys. Rev. Lett., 1987, 58, 2087; doi 10.1103/PhysRevLett.58.2087

8. Kardar M., Nucl. Phys. B, 1987, 290, 582; doi 10.1016/0550-3213(87)90203-3

9. Bouchaud J.P., Orland H., J. Stat. Phys., 1990, 61, 877; doi 10.1007/BF01027306

10. Brunet E., Derrida B., Phys. Rev. E, 2000, 61, 6789; doi 10.1103/PhysRevE.61.6789

11. Johansson K., Commun. Math. Phys., 2000, 209, 437; doi 10.1007/s002200050027.

12. Prähofer M., Spohn H., J. Stat. Phys., 2002, 108, 1071; doi 10.1023/A:1019791415147

13. Ferrari P.L., Spohn H., Commun. Math. Phys., 2006, 265, 1; doi 10.1007/s00220-006-1549-0

14. Sasamoto T., Spohn H., Phys. Rev. Lett., 2010, 104, 230602; doi 10.1103/PhysRevLett.104.230602

15. Sasamoto T., Spohn H., Nucl. Phys. B, 2010, 834, 523; doi $10.1016 /$ j.nuclphysb.2010.03.026

16. Sasamoto T., Spohn H., J. Stat. Phys., 2010, 140, 209; doi 10.1007/s10955-010-9990-z.

17. Amir G., Corwin I., Quastel J., Commun. Pure Appl. Math., 2011, 64, 466; doi 10.1002/cpa.20347

18. Dotsenko V., Klumov B., J. Stat. Mech., 2010, P03022; doi 10.1088/1742-5468/2010/03/P03022

19. Dotsenko V., Europhys. Lett., 2010, 90, 20003; doi 10.1209/0295-5075/90/20003

20. Dotsenko V., J. Stat. Mech., 2010, P07010; doi 10.1088/1742-5468/2010/07/P07010

21. Calabrese P., Le Doussal P., Rosso A., Europhys. Lett., 2010, 90, 20002; doi $10.1209 / 0295-5075 / 90 / 20002$

22. Calabrese P., Le Doussal P., Phys. Rev. Lett., 2011, 106, 250603; doi 10.1103/PhysRevLett.106.250603

23. Le Doussal P., Calabrese P., J. Stat. Mech., 2012, P06001; doi 10.1088/1742-5468/2012/06/P06001 Preprint arXiv:1204.2607 2012.

24. Dotsenko V., J. Stat. Mech., 2012, P11014; doi 10.1088/1742-5468/2012/11/P11014

25. Gueudré T., Le Doussal P., Europhys. Lett., 2012, 100, 26006; doi 10.1209/0295-5075/100/26006

26. Corwin I., Random Matrices: Theory Appl., 2012, 1, 1130001; doi 10.1142/S2010326311300014. Preprint arXiv:1106.1596 2011.

27. Borodin A., Corwin I., Ferrari P., Preprint arXiv:1204.1024 2012.

28. Tracy C.A., Widom H., Commun. Math. Phys., 1994, 159, 151; doi 10.1007/BF02100489

29. Prolhac S., Spohn H., J. Stat. Mech., 2011, P01031; doi 10.1088/1742-5468/2011/01/P01031.

30. Dotsenko V., J. Phys. A: Math. Theor., 2013, 46, 355001; doi 10.1088/1751-8113/46/35/355001

31. Imamura T., Sasamoto T., Spohn H., J. Phys. A: Math. Theor., 2013, 46, 355002; doi $10.1088 / 1751-8113 / 46 / 35 / 355002$

32. Prolhac S., Spohn H., J. Stat. Mech., 2011, P03020; doi 10.1088/1742-5468/2011/03/P03020

33. Lieb E.H., Liniger W., Phys. Rev., 1963, 130, 1605; doi 10.1103/PhysRev.130.1605

34. McGuire J.B., J. Math. Phys., 1964, 5, 622; doi 10.1063/1.1704156

35. Yang C.N., Phys. Rev., 1968, 168, 1920; doi 10.1103/PhysRev.168.1920

36. Calabrese P., Caux J.-S., Phys. Rev. Lett., 2007, 98, 150403; doi/10.1103/PhysRevLett.98.150403

37. Dotsenko V.S., Physics-Uspekhi, 2011, 54, No. 3, 259; doi 10.3367/UFNe.0181.201103b.0269

38. Dotsenko V., J. Stat. Mech., 2013, P02012; doi 10.1088/1742-5468/2013/02/P02012 


\section{$N$-точкова функція розподілу вільної енергії в одновимірних хаотично напрямлених полімерах}

В. Доценко112

1 Університет м. Париж VI, 75252 Париж, Франція

2 Інститут теоретичної фізики ім. Л.Д. Ландау, 119334 Москва, РФ

Отримано явний вираз для $N$-точкової функції розподілу вільної енергії в одновимірному напрямленому полімері в термінах анзацу Бете в рамках методу реплік. Отриманий результат еквівалентний результату, раніше отриманому в роботі Пролака і Шпона [J. Stat. Mech., 2011, P03020].

Ключові слова: напрямлені полімери, хаотичний потенціал, репліки, флуктуації, функція розподілу 